\title{
Structure- Fluisd Coupling for Micro fluidic applications
}

\author{
Houaria Bourbaba ${ }^{1}$, Chellali Benachaiba ${ }^{1}$ \\ ${ }^{1}$ physics of Semiconductors Devices Laboratory, Bechar University, Bechar, algeria
}

\begin{abstract}
The polymer materials have been proposed to be good candidates for the development of the microfluidic devices, in this paper the structure fluid coupling in micro fluidic actuations is investigated. A miniature actuator consists of a PDMS elastomeric membrane and PMMA canalisations were modeled by using finite element programmers. We used hyper elastic models to model the mechanical behavior of the membrane under pressure, this type of modeling can provide a better understanding of deformation mechanisms, the deformation of the membrane can be used to push a fluid in a canalization contact with the membrane. As a second step, we have coupled between the mechanical behavior of the membrane and the fluid flow in the canalization.
\end{abstract}

Keywords - Finite element, hyperelastic, modeling, PDMS, structure fluid coupling.

\section{INTRODUCTION}

The study of the mechanical behavior of the polymer materials made the object of numerous studies, and constitutes another rich domain of research. In particular there is a request for rapid techniques of prototyping for making micro fluidic devices endowed with a greater complexity, of shorter time of manufacturing, lower costs.

The use of elastomers of PolyDiMethylSiloxane (PDMS) is the most common law for manufactured Microfluidic devices [1-2]. The devices are finalized by closure of structures by gluing on flat surface (glass, elastomers, ....). In particular the PDMS is very simple to manipulate and permits to retort perfectly by milling of the micrometric motives. In addition to the simplicity of manipulation, the PDMS has the following properties: it is a hyperelastic polymer; it can undergo very big distortions therefore without deteriorating [3]. It is biocompatible and non porous to the liquids. The PDMS is the main support for the manufacture of fluidic Microsystems. The PDMS used in our work is silgel 612 [4], it is the standard material for the applications micro fluidics.

Our goal is to study the mechanical behavior of elastomeric membrane using a hyperelastic model of NeoHookean and compare the results with the linear model for small deformations.

Modeling and simulation of the system fluid structure are the key steps in the design and optimization of the micro fluidic applications. The description of the mechanical behavior of the elastomer and displacement of fluid generated by the membrane deformation are investigated by coupling between structure model and fluid model.

\subsection{Mechanical Behavior}

\section{Modelling}

\subsubsection{Hyper-elastic Model}

A hyperelastic material is incompressible present a big deformation, the stress-strain relationship derives from a strain energy density function [5].

In continuum mechanics large strain elastic (hyperelastic) materials are characterized through a strain energy potential $W$, which represents the strain energy of the material as a function of the deformation. In the NeoHookean model the strain energy density function for PDMS, $W$ expressed as:

$W=\frac{1}{2} G\left(l_{1}-3\right)+\frac{1}{2} K(y-1)^{2}$

Where:

$I_{l}$ is the strain invariant: $I_{I}=\operatorname{tr} C$

$J=\operatorname{det}(F)$

$F$ is the deformation tensor.

$C$ is Cauchy-Green tensor: $C=F^{T} F$

$I$ is the second-order unit tensor.

$\varepsilon$ is the Green strain tensor: $\varepsilon=\frac{1}{2}(C-D)$

The second Piola-Kirchhoff stress tensor can be expressed as

$s=\partial W / \partial \epsilon$

The Cauchy stress tensor can be expressed as [6]: 
$\sigma=\frac{1}{j} F S F^{T}$

The shear modulus $G$ and the bulk modulus $K$ of the hyper elastic material are defined by the following relations:

$G=\frac{E}{2(1+v)}$
$K=\frac{E}{3(1-2 v)}$

Where $E$ is Young's modulus and $v$ is t Poisson's ratio.

\subsubsection{Linear Elastic Model}

Under small strain the deformation of PDMS membrane are very little. We can use the linear model for the first order approximation.

The linear elastic model is based on Hooke's law. For isotropic model, the behavior of the material is governed by two material parameters. There are four material parameters for an elastic model, the elastic modulus $E$, Poisson's ratio $v$, bulk modulus $K$ and shear modulus $G$.

The general form of Hooke's law for isotropic materials may now be written as a linear combination of strain and stress tensors:

$\sigma_{i j}=3 K\left(\frac{1}{3} \epsilon_{\mathrm{kh}} \delta_{i j}\right)+2 G\left(\epsilon_{\mathrm{i} j}-\frac{1}{3} \epsilon_{\mathrm{kh}} \delta_{i j}\right)$

Where $\delta_{i j}$ is the Kronecker Delta

\subsection{Fluid Model}

As a second step we modeled the behavior of the fluid of membrane. In fluid mechanics the equations correspond to a flow Eulerian description. In such description defines a flow velocity of the fluid in any point of the repository. This description opposes the Lagrangian description that is to follow the particles in their movement and therefore sets the movement of objects by their position at any moment. This method is better suited to the mechanics of the solid [7].For a Newtonian (water in this case) fluid, the fluid can be considered incompressible, the equation of fluid dynamics that describes the movement of the water is the Navier-Stokes equation:

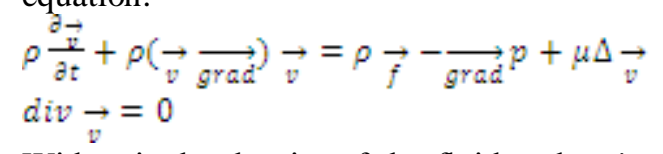

With $\rho$ is the density of the fluid and $\rightarrow$ is the velocity of a fluid element, $\rho \rightarrow \vec{f}$ represents the set of volume forces applied to the fluid, $\mathrm{p}$ the pressure and $\mu$ the viscosity of the fluid.

\section{NUMIRICAL SIMULATION}

The general purpose FE program has been used for the present calculations. The 3D model for the analysis square membrane $(800 \mu \mathrm{m} \times 800 \mu \mathrm{m} \times 20 \mu \mathrm{m})$ is shown in fig. 1 .

the simulation used for quart of membrane to decrease the time of calculation. The membrane loaded by pressure tension at center in z-direction and boundary conditions used in simulation is indicated. The table 1 presents the elastic parameters of the PDMS [4].

Table 1: PDMS parameters

\begin{tabular}{|c|c|c|}
\hline $\begin{array}{l}\text { ELASTIC } \\
\text { PARAMETERS }\end{array}$ & $E(\mathrm{MPA})$ & $v$ \\
\hline \multicolumn{1}{|c|}{ PDMS } & 4 & 0.48 \\
\hline
\end{tabular}




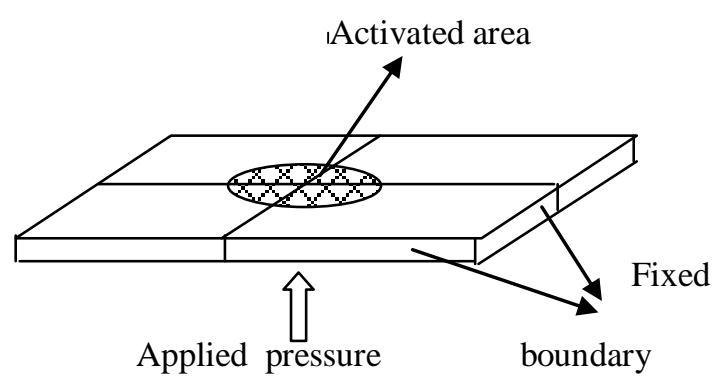

Fig.1 the model of membrane and boundary conditions

In the second part, to model the fluid membrane (fig. 2) contact we used a hybrid formulation, called ALE "Arbitrary Lagrangian Eulerian" formulation, this method allows to pass in a continuous way of spatial coordinates in the material coordinates and vice versa [8].

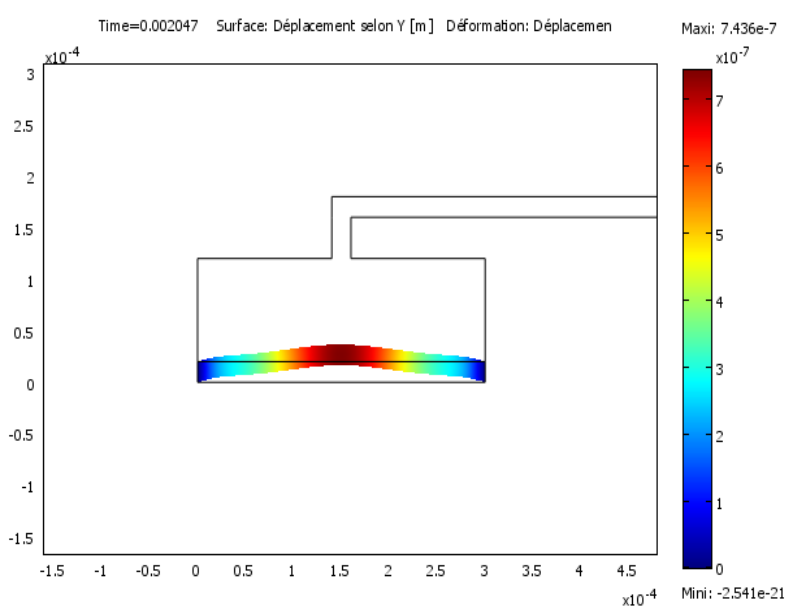

Fig.2 Fluid-membrane contact

\section{RESULTS AND DISCUSSIONS}

The membrane of PDMS will deform under applied pressure, as shown in Fig.3. To compare between the two models, the $\mathrm{NH}$ and linear model are used to simulate the deformation. The vertical displacements on the surface of PDMS membrane are shown in Fig.4, from these curves we found that the NH and linear model gives similar results in the case of small strain. That we can use the linear model rather than more complicates $\mathrm{NH}$ model to solve such a problem.

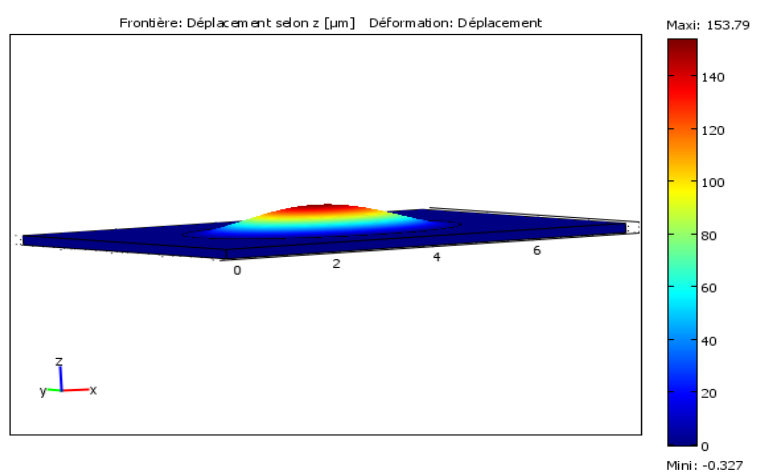

Fig. 3 Deformation of PDMS membrane induced by applied Pressure 


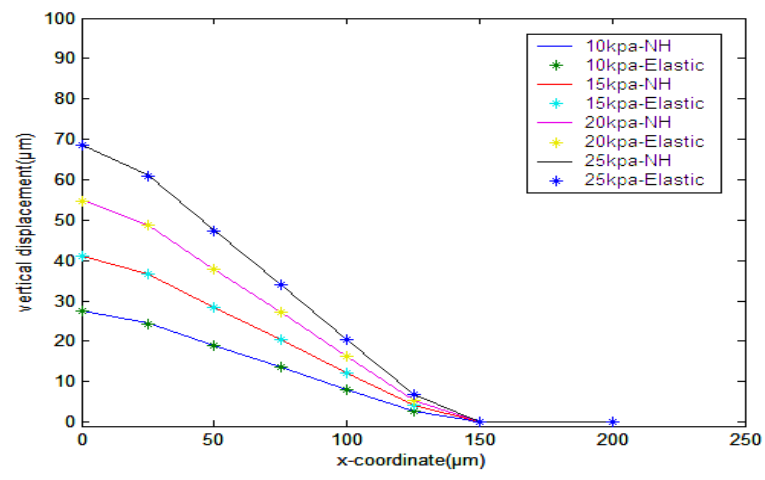

Fig.4 Vertical displacement on surface of PDMS membrane at different pressure.

As shown in Fig.5, the maximum of vertical displacement increase with increasing applied pressure, and the PDMS membrane has a large deformation when we increase the applied pressure.

In goal to study the effect of the membrane dimensions on the rigidity of system, the response of the membrane is tested in function of the membrane thickness (Fig.6). We not that the deformation of the membrane decrease with increasing thickness values, so we interest to minimize the thickness of membrane to get the lager deformation with smaller applied pressure.

The membrane deformation pushed the fluid (water) in the canalization (Fig. 7), the simulation of the system structure fluid contact allowed us to estimate the value of fluid speed in canalization.

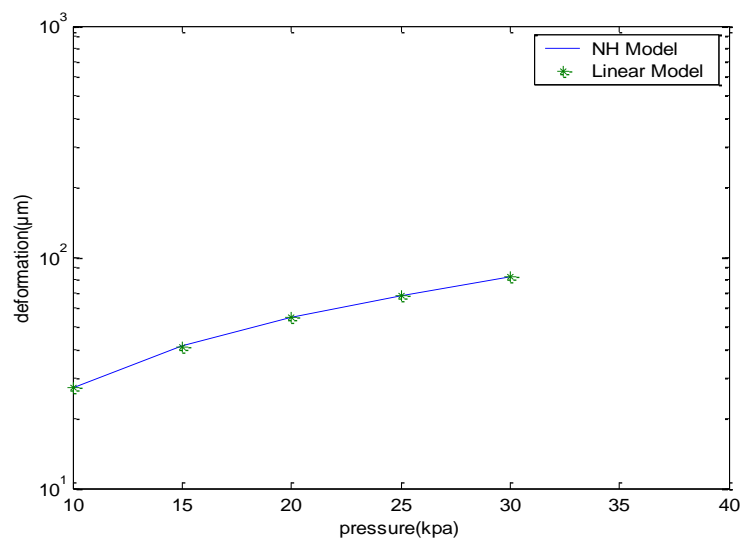

Fig.5 Maximum of vertical displacement on surface of PDMS membrane at different pressure

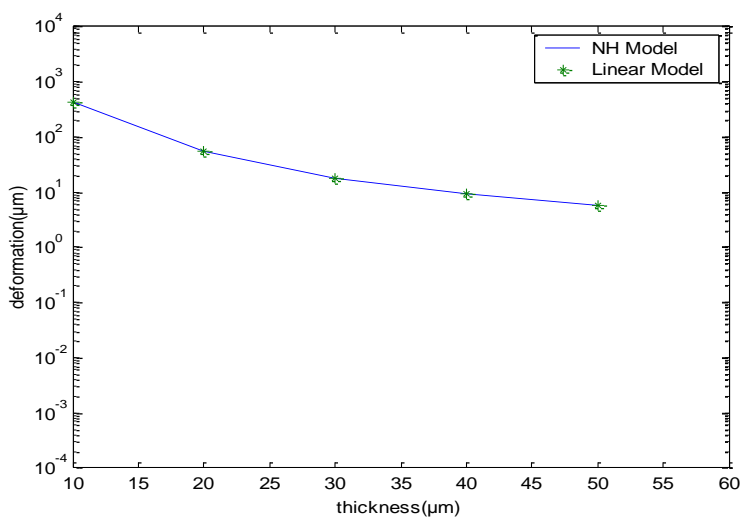

Fig.6 Maximum of vertical displacement on surface of PDMS and PMMA membrane at different thickness. 


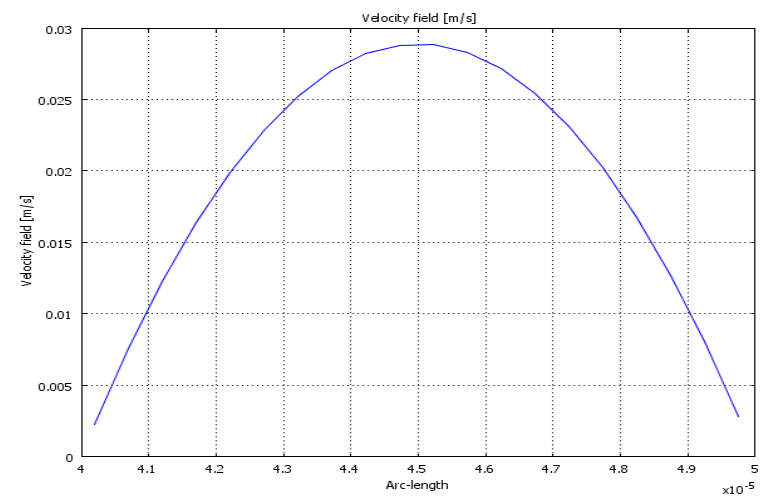

Fig. 7 field velocity in canalization.

\subsection{Mesh Tests}

To optimize the response of deformation membrane, the results of simulation by Finite element are tested for different values of elements and are summarized in table 2 .

Table 2: The tested mesh for PDMS membrane deformation at 10kpa

\begin{tabular}{|c|c|c|c|c|}
\hline $\begin{array}{c}\text { ELEMENT } \\
\text { NUMBERS }\end{array}$ & 1271 & 3754 & 10611 & 14214 \\
\hline $\begin{array}{c}\text { MAXIMUM } \\
\text { DEFORMATION } \\
(\mu \mathrm{M})\end{array}$ & 28.13 & 29.22 & 30.89 & 31.21 \\
\hline $\begin{array}{c}\text { CALCULATED } \\
\text { TIME }(\mathrm{S})\end{array}$ & 1.015 & 2.156 & 6.469 & 29.203 \\
\hline $\begin{array}{c}\text { RELATIVE } \\
\text { ERROR }(\%)\end{array}$ & 9.86 & 6.37 & 1.02 & 0 \\
\hline
\end{tabular}

We notice that relative error is less than $10 \%$ therefore we choose the smallest number of element to optimize the time of calculates.

\section{CONCLUSION}

The mechanical answer of a membrane polymeric is studied one uses a linear model and a hyperelastic model using Finite Element program. We have showed that the results of simulation gotten by the two models are similar for small values of pressure. In this case we can replace the hyperelastic model by the simple linear model.

The deformation of PDMS for different values of pressure and thickness show that: the maximum of vertical displacement increase with pressure and reversely decrease with increasing thickness. the mechanical response of PDMS membrane, show that the it is better for microfluidics applications used as active membrane, where we need small applied pressure to have a large deformation.

An applied pressure generate deformation of structure actuator, where we can induce displacement of fluide in canalisation (Fig.2). the modeling of a fluid structur interaction We allow to calculate the speed of the fluid in the canalization. This model is the principle of the microvalve which has several fields of application.

\section{REFERENCES}

[1] C.H. Wang, G.B. Lee, Biosens. Bioelectron 21(2005) 419-293.

[2] A. Mata, A.J. Fleischman, S.Roy, Characterization of polydimethylsiloxane (PDMS) properties for biomedical micro/nanosystems, Biomed. Microdevice 7(2005) 281-293.

[3] M. Unger, H. Chou, T. Thorsen, A. Sherer, and S. Quake, Monolithic microfabricated valves and pumps by multilayer soft lithography, Science 288 (5463):113-116-(2000),

[4] WACKER, Technical data sheet for WACKER SilGel® 612 A/B, 2008.

[5] R.W. Ogden, Non -linear Elastic Deformation, Dover, New York,1997.

[6] H. Daanouni, Y. Tillier, F. Bay, numeric survey Of hyperelastic membrane subjected to muliaxial loadings, 19 French Congres of Mechanics, 2009, Marseille.

[7] A. Hoel, Conception et analyse d'un microsystème pour l'injection ransdermique, doctoral diss, Teacher's Training College Superieure of Cachan,France 2007.

[8] Cadiou A., NadiaLES, Manuel théorique,Note technique CODICIEL-LMFA, N_2003-01, 